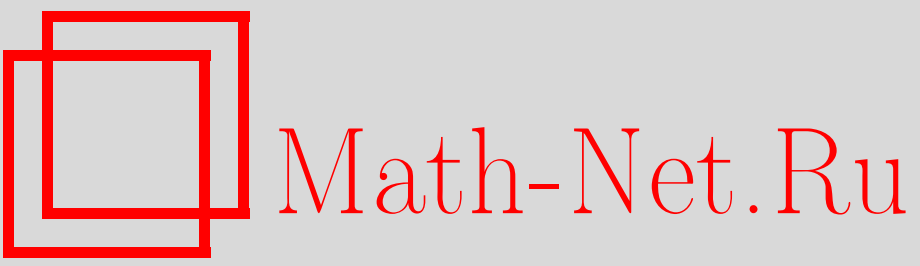

Ф. Ф. Воронов, Исчисление Картана для дуальных форм, УМН, 2001, том 56, выпуск 2, 211-212

DOI: https://doi.org/10.4213/rm387

Использование Общероссийского математического портала Math-Net.Ru подразумевает, что вы прочитали и согласны с пользовательским соглашением

http://www . mathnet.ru/rus/agreement

Параметры загрузки:

IP : 54.198 .64 .247

26 апреля 2023 г., $16: 27: 33$ 


\title{
ИСЧИСЛЕНИЕ КАРТАНА ДЛЯ ДУАЛЬНЫХ ФОРМ
}

\author{
Ф. $\Phi$. Воронов
}

1. Дуальные и смешанные формы. Рассмотрим суперпространство $V=V_{0} \oplus V_{1}$ размерности $\operatorname{dim} V=n \mid m$ (которое мы отождествляем с линейньм супермногообразием). Пусть Vol $V$ обозначает пространство форм объема на $V$. Гладкое отображение

$$
\mathscr{L}: \underbrace{V^{*} \times \cdots \times V^{*}}_{p} \times \underbrace{V^{*} \Pi \times \cdots \times V^{*} \Pi}_{q} \rightarrow \operatorname{Vol} V
$$

есть дуальная форма на $V$ костепени $p \mid q$, если выполнены условия: $\mathscr{L}(p h)=\mathscr{L}(p) \operatorname{Ber} h$, для всех $h \in \mathrm{GL}(p \mid q)$, и

$$
\frac{\partial^{2} \mathscr{L}}{\partial p_{A}{ }^{K}} \partial p_{B}{ }^{L}+(-1)^{\widetilde{A} \widetilde{B}+(\widetilde{A}+\widetilde{B}) \widetilde{L}} \frac{\partial^{2} \mathscr{L}}{\partial p_{B}{ }^{K}} \partial p_{A}{ }^{L}=0
$$

Аргументы $\mathscr{L}$ (ковекторы) пишутся в столбик, и они образуют матрицу $p=\left(p_{A}{ }^{K}\right)$. В силу первого условия, нечетные ковекторы $p^{K}, \widetilde{K}=1$, должны быть линейно независимы, откуда $0 \leqslant q \leqslant m$

Рассмотрим теперь $V \oplus \mathbb{R}^{r \mid s}$ для некоторой размерности $r \mid s$. Обозначим $W:=V \oplus \mathbb{R}^{r \mid s}$. Смешанная форма [1] на $V$ - это гладкое отображение

$$
\mathscr{L}: \underbrace{W^{*} \times \cdots \times W^{*}}_{p} \times \underbrace{W^{*} \Pi \times \cdots \times W^{*} \Pi}_{q} \rightarrow \operatorname{Vol} V,
$$

удовлетворяющее условиям $\mathscr{L}(p h, w h)=\mathscr{L}(p, w) \operatorname{Ber} h$ для всех $h \in \mathrm{GL}(p \mid q), \mathscr{L}(p+a w, g w)=$ $\mathscr{L}(p, w)$ Ber $g$, для всех $g \in \operatorname{GL}(r \mid s)$ и всех $a \in \operatorname{Mat}(r|s \times n| m)$, и уравнениям, аналогичным (1), для всей совокупности переменных $p=\left(p_{A}{ }^{K}\right), w=\left(w_{F}{ }^{L}\right) ; p \mid q$ есть костепень, $r \mid s$ дополнительная степень. Для данного $K$ матричные элементы $p_{A}{ }^{K}, w_{F}{ }^{K}$ суть компоненты ковектора на $V \oplus \mathbb{R}^{r \mid s}$. Отметим, что $s \leqslant q \leqslant m+s$ из-за условий с березинианом. Примеры дуалшных и смешанных форм строятся как и для "прямых" форм [1]-[3].

Введем обозначения $\Lambda_{p \mid q}=\Lambda_{p \mid q}(V)$ и $\Lambda_{p \mid q}^{r \mid s}=\Lambda_{p \mid q}^{r \mid s}(V)$ для пространств дуальных и смешанных форм соответственно. Заметим, что $\Lambda_{p \mid q}=\Lambda_{p \mid q}^{0 \mid 0}$. Имеет место изоморфизм $\tau: \Lambda^{r \mid s} \rightarrow$ $\Lambda_{n \mid m}^{r \mid s}$ с пространством прямых форм [1]: $L=L(v) \mapsto \mathscr{L}=\mathscr{L}(p, w)$, где $\mathscr{L}(p, w):=$ $L\left(w p^{-1}\right)$ Ber $p$. Имеют место [1] изоморфизмы стабильности: $\sigma_{k \mid l}: \Lambda_{p \mid q}^{r \mid s} \rightarrow \Lambda_{p+k \mid q+l}^{r+k \mid s+l}$, где

$$
\left(\sigma_{k \mid l} \mathscr{L}\right)\left(\begin{array}{cc}
p_{1} & p_{2} \\
w_{11} & w_{12} \\
w_{21} & w_{22}
\end{array}\right):=\mathscr{L}\left(\begin{array}{c}
p_{1}-p_{2} w_{22}{ }^{-1} w_{21} \\
w_{11}-w_{12} w_{22}{ }^{-1} w_{21}
\end{array}\right) \cdot \operatorname{Ber} w_{22}
$$

Разбиение матрицы в аргументе на блоки отвечает выделению последних $k \mid l$ строчек и столбцов. Обратньй изоморфизм $\sigma_{k \mid l}^{-1}$ задается подстановкой $p_{1}:=p, w_{11}:=w, w_{22}:=1, p_{2}:=0$, $w_{12}:=0, w_{21}:=0$. Можно говорить о прямом пределе $\Lambda^{r \mid s}(V):=\underline{\lim }_{N, M} \Lambda_{n+N \mid m+M}^{r+N \mid s+M}(V)$, где $-\infty<r<+\infty, 0 \leqslant s \leqslant m$. В частности, для любого $r \geqslant 0 \boldsymbol{\Lambda}^{r \mid s}(V) \cong \Lambda^{r \mid s}(V)$, и для любого $r \leqslant n \Lambda^{r \mid s}(V) \cong \Lambda_{n-r \mid m-s}(V)$.

Работа выполнена при частичной поддержке EPSRC, грант GR/N00821/01. 
2. Операции $e(\alpha)$ и $e(u)$. Для $\alpha=e^{A} \alpha_{A} \in V^{*}, u=u^{A} e_{A} \in V$ определим $e(\alpha)$ : $\Lambda_{p \mid q}^{r \mid s} \rightarrow \Lambda_{p \mid q}^{r+1 \mid s}$ и $e(u): \Lambda_{p \mid q}^{r \mid s} \rightarrow \Lambda_{p+1 \mid q}^{r \mid s}$ формулами: $e(\alpha) \mathscr{L}:=(-1)^{r} \alpha_{A} w_{r+1}^{K}(-1)^{\widetilde{\alpha} \widetilde{A}} \frac{\partial \mathscr{L}}{\partial p_{A} K}$, $e(u) \mathscr{L}:=(-1)^{r} u^{A}\left(p_{A}^{p+1}-(-1)^{\widetilde{B} \widetilde{K}} p_{A}{ }^{K} p_{B}^{p+1} \frac{\partial}{p_{B} K}-(-1)^{\widetilde{F} \widetilde{K}} p_{A}{ }^{K} w_{F}^{p+1} \frac{\partial}{w_{F}^{K}}\right) \mathscr{L}$.

Tеорема 1. Операции е( $\alpha), e(u)$ стабильны. Они удовлетворяют антикоммутационным соотношениям: $e(u) e(v)+(-1)^{\widetilde{u} \widetilde{v}} e(v) e(u)=0, e(\alpha) e(\beta)+(-1)^{\widetilde{\alpha} \widetilde{\beta}} e(\beta) e(\alpha)=0$, $e(u) e(\alpha)+(-1)^{\widetilde{\alpha} \widetilde{u}} e(\alpha) e(u)=\langle u, \alpha\rangle \sigma$, əдe $u, v \in V, \alpha, \beta \in V^{*}, u \sigma=\sigma_{1 \mid 0}: \Lambda_{p \mid q}^{r \mid s} \rightarrow \Lambda_{p+1 \mid q}^{r+1 \mid s}$. Для любого $r$ при изоморфизме $\tau$ e $(\alpha)$ переходит на прямых формах во внешнее умножение на $\alpha, e_{\alpha}: \Lambda^{r \mid s} \rightarrow \Lambda^{r+1 \mid s}$. Для $r>0$ операция $е(u)$ переходит в свертку с и: $\tau^{-1} \circ \sigma_{1 \mid 0}^{-1} \circ e(u) \circ \tau=i_{u}: \Lambda^{r \mid s} \rightarrow \Lambda^{r-1 \mid s}$. Для $r=0$ получаем отображение $\sigma_{0 \mid s}^{-1} \circ e(u) \circ \tau: \Lambda^{0 \mid s} \rightarrow \Lambda_{n+1 \mid m-s}$, “исправляющее” операцию $i_{u}$. Подобное верно и для дуальных форм.

Из теоремы 1, в частности, вытекает, что смешанные формы образуют модул над "четной" версией внешней алгебры, $\Lambda\left(V^{*}\right):=T\left(V^{*}\right) /\left(\alpha \otimes \beta+(-1)^{\widetilde{\alpha}} \widetilde{\beta} \beta \otimes\right)$.

3. Тождество гомотопии. Рассмотрим супермногообразие $M=M^{n \mid m}$ и пучки смешанных форм $\Omega_{p \mid q}^{r \mid s}$. "Вариационный” дифференциал действует на смешанные формы по формуле [1]: $d \mathscr{L}:=(-1)^{r} w_{r+1}^{K}(-1)^{\widetilde{A} \widetilde{K}} \frac{\partial}{\partial x^{A}} \frac{\partial \mathscr{L}}{\partial p_{A}^{K}}$. Он увеличивает степень на единицу. Пусть $u-$ векторное поле на $M$. Обычным образом определяется производная Ли. Дифференциал и производная Ли стабильны. Явная формула для производной Ли:

$$
\delta_{u} \mathscr{L}=u^{A} \frac{\partial \mathscr{L}}{x^{A}}-(-1)^{\widetilde{A} \widetilde{X}} \frac{\partial u^{B}}{\partial x^{A}} p_{B} K \frac{\partial \mathscr{L}}{\partial p_{A} K}+(-1)^{\widetilde{A}(\widetilde{X}+1)} \frac{\partial u^{A}}{\partial x^{A}} \mathscr{L} .
$$

Лемма. $d(f \mathscr{L})=f d \mathscr{L}+e(d f) \mathscr{L}$, для любой функции $f$.

По теореме 1 , определен оператор умножения форм из $\Omega_{p \mid q}^{r \mid s}$ на элементы $\Omega^{*}=\bigoplus \Omega^{k}$ ("наивный” комплекс де Рама с четным дифференциалом, см. [4]). Обозначаем его, как и выше, $e(\omega)$.

Теорема 2. Имеют место соотношения

$$
\begin{aligned}
đ e(\omega)-(-1)^{k} e(\omega) d & =e(d \omega) & & \text { (тождество Лейбница), } \\
đ e(u)+e(u) d & =\sigma \delta_{u} & & \text { ("тождество гомотопии"), }
\end{aligned}
$$

для любой $\omega \in \Omega^{k}$ и любого векторного поля $u$.

В стабильном пределе из (4) вытекает обычная форма тождества гомотопии. Равенство (4) может быть доказано непосредственной вькладкой для композиции $\sigma^{-1}$ с левой частью.

\section{СПИСОК ЛИТЕРАТУРЫ}

[1] Th. Voronov. Supermanifold forms and integration. A dual theory // Solitons, Geometry, and Topology: On the Crossroad / ed. V.M. Buchstaber, S. P. Novikov. Providence, RI: Amer. Math. Soc., 1997. P. 153-171. (Amer. Math. Soc. Transl. Ser. 2. V. 179 (33).); dg-ga/9603009. [2] O. M. Khudaverdian. Batalin-Vilkovisky formalism and odd symplectic geometry // Proceedings of the Workshop "Geometry and Integrable Models", Dubna, 4-8 Oct. 1994. Singapore: World Scientific, 1995; hep-th 9508174. [3] Th. Voronov. Geometric integration theory on supermanifolds. V. 9, 1992. [4] Ю. И. Манин. Калибровочные поля и комплексная геометрия. М.: Наука, 1984.

University of Manchester Institute of Science and Technology (UMIST)

E-mail: theodore.voronov@umist.ac.uk 